\title{
An Intelligent Fleet Condition-Based Maintenance Decision Making Method Based on Multi-Agent
}

\author{
Qiang Feng ${ }^{1}$, Songjie $\mathrm{Li}^{2}$, and $\mathrm{Bo} \mathrm{Sun}^{3}$ \\ ${ }^{1,2,3}$ School of Reliability and Systems Engineering, Beihang University, Beijing, 100191, China \\ fengqiang@buaa.edu.cn \\ songjieli@dse.buaa.edu.cn \\ sunbo@buaa.edu.cn
}

\begin{abstract}
According to the demand for condition-based maintenance online decision making among a mission oriented fleet, an intelligent maintenance decision making method based on Multi-agent and heuristic rules is proposed. The process of condition-based maintenance within an aircraft fleet (each containing one or more Line Replaceable Modules) based on multiple maintenance thresholds is analyzed. Then the process is abstracted into a Multi-Agent Model, a 2-layer model structure containing host negotiation and independent negotiation is established, and the heuristic rules applied to global and local maintenance decision making is proposed. Based on Contract Net Protocol and the heuristic rules, the maintenance decision making algorithm is put forward. Finally, a fleet consisting of 10 aircrafts on a 3-wave continuous mission is illustrated to verify this method. Simulation results indicate that this method can improve the availability of the fleet, meet mission demands, rationalize the utilization of support resources and provide support for online maintenance decision making among a mission oriented fleet.
\end{abstract}

\section{INTRODUCTION}

When conducting a mission, an aircraft fleet consumes massive resources, especially maintenance manpower and resources. In practice, maintenance strategies usually combine the "fail and fix maintenance" with fixed preventive maintenance. The "fail and fix" strategy cannot prevent fatal accidents, which may endanger pilots' lives and reduce the mission availability, while fixed preventive maintenance strategy usually schedules excess maintenance actions to ensure availability, while ignoring the asynchronism of failures among a fleet and the shareability of maintenance resources, hence, cannot fully develop the

Qiang Feng et al. This is an open-access article distributed under the terms of the Creative Commons Attribution 3.0 United States License, which permits unrestricted use, distribution, and reproduction in any medium, provided the original author and source are credited. overall efficiency of maintenance resources, causing a huge waste while cannot completely prevent failure (Jiang \& Murthy, 2008). Besides, to ensure safety, a specific maintenance job is done at a specific site, which may lead to the incoordination between operational requirements and maintenance actions. In general, traditional "fail and fix" practice \& fixed preventive maintenance practice are not completely suitable.

To tackle the difficult problem, Condition-Based Maintenance (CBM), which is based on the actual condition and development tendency of assets, is put forward (Bengtsson, 2004). The rapid development of Prognostics and Health Management (PHM) (Sun, Zeng, Kang \& Pecht, 2012) approach and its application on battery (Goebel, Saha, Saxena, Celaya \& Christophersen, 2008) and aero engine (Wen \& Liu, 2011) makes $C B M$ possible. In practice, an aircraft contains one or more Line Replaceable Modules $(L R M)$ whose health condition development fit the deterioration process (Barata, Guedes, Marseguerra \& Zio., 2002). PHM can help predict the Residual Useful Life (RUL) of deteriorating $L R M s$ through condition monitoring, and help staff make maintenance decision. Through the application of $P H M$, a series of maintenance measures are provided in time, and the ideal $C B M$ "need and fix" is achieved (Jardine, Lin \& Banjevic, 2006). Moreover, since $R U L$ can be estimated, maintenance actions can be performed dynamically according to operational requirements rather than in a fixed site. In a fleet, where maintenance tasks are heavy and resources are limited, the application of $C B M$ can notably increase operational availability, reduce lifecycle costs and improve safety.

Traditional $C B M$ is about safely extending maintenance intervals using $P H M$ information, and is often applied to a single aircraft. Fleet oriented $C B M$, on the other hand, should consider many factors other than single aircraft $C B M$, such as mission requirement, maintenance teams, etc., to balance the whole fleet. Actually, the ideal process of fleet $C B M$ is as follows: 1) Aircrafts obtain their PHM data. 2) The PHM data is transferred to the maintenance center. 3) 
The maintenance center makes maintenance decisions. 4) The maintenance decisions are transferred to aircrafts and maintenance teams. 5) Maintenance action. So the fleet $C B M$ problem is actually an "online" decision making problem. Besides, the fleet maintenance strategy is the combination of maintenance strategies for every single aircraft. For each single aircraft, the problem is to find the most suitable time and team while balancing the whole fleet, which is actually a routing problem. Routing problem has already proved to be N-P hard (Garey \& Johnson. 1979), which is difficult to obtain the optimal or satisfying solution with the increase of problem scale. At present, the main solutions to fleet $C B M$ problem include

1. Mathematical programming: Doganay and Bohlin (2010) studied the train fleet maintenance scheduling strategy \& spare parts optimization with single station based on a mixed integer linear programming. Bai (2009) optimized the Life Limited Part (LLP) group maintenance schedule and the on-wing lifetime of an aero engine fleet based on immune particle swarm method.

2. Heuristic method: Reimann, Kacprzynski, Cabral and Marini (2009) designed a maintenance scheduling algorithm combining $C B M$ with traditional fixed preventive maintenance using heuristic method, to reduce the maintenance cost of a fleet consisting of 50 aircrafts, and to predict the shortage of maintenance resources.

3. System simulation: Bivona and Montemaggiore (2005) tested different maintenance \& management strategies based on system dynamics modeling and simulation. Dupuy, Wesely and Jenkins (2011) selected the best one out of three civil aviation fleet maintenance plans applying discrete event simulation with the help of ARENA ${ }^{\circledR}$.

4. Artificial Intelligence: Cycon (2011) discussed the technique Sikorsky Aircraft Corporation (SAC) is applying to incorporate $C B M$ capabilities into all its products. By data collecting from all products and data mining, normal versus anomalous behavior is established, and man-in-the-loop support allows experts from various engineering and support services groups to quickly recommend appropriate maintenance actions. Zhou, Fox, Lee and Nee (2004) applied Multi-Agent technique and heuristic rules to solve the bus maintenance scheduling problem, which has equal optimality to reported studies and requires less computing time.

5. Multiple criteria analysis: Papakostas, Papachatzakis, Xanthakis, Mourtzis and Chryssolouris (2010) applied the multiple criteria (Cost, RUL, Operational Risk \& Flight Delay) based on specific mission to select the best out of a set of generated maintenance plan alternatives using Monte-Carlo simulation.
But there are still shortages between those methods and dynamic environments where online maintenance decision making and scheduling is required when an aircraft fleet execute combat tasks. Especially in:

1. Those methods lack consideration into the relationship between the health condition of the entire fleet and that of a single aircraft, ignoring the potential shortage of maintenance resources, and the maintenance scheduling strategy is usually not optimal.

2. Due to the uncertainty of tasks and variety of aircrafts' health condition, maintenance strategy needs to be generated according to mission demands, aircrafts' health condition and resource limits. Those methods lack consideration into online decision making.

The fleet maintenance problem involves a lot of communication among aircrafts and maintenance teams, and Multi-Agent Modeling technique can imitate the communication and cooperation among agents to model complex systems (Budenske, Newhouse, Bonney \& Wu, 2001), and has been successfully applied in many fields of manufacturing, especially dynamic and distributed scheduling problems. Through communication and cooperation can aircrafts and maintenance teams acquire the health condition of the whole fleet, and the working condition of maintenance teams. Meanwhile, the fleet maintenance problem is an N-P hard problem, and a common solution to N-P hard problems is heuristic searching. Heuristic rules can be integrated into agents to help overcome the N-P hardness, and is a guide to the intelligent allocation of maintenance tasks (Yang \& Hu, 2007). In one word, Multi-Agent Modeling is suitable for solving the aircraft fleet maintenance problem.

This paper is the application of Multi-Agent System (MAS) to aircraft fleet maintenance scheduling. In this article, the idea of $M A S$ and heuristic rules is adopted, and the dynamic intelligent maintenance decision making among an aircraft fleet with multiple maintenance teams is achieved to provide technical support for the online maintenance decision making. The purpose of this paper is to propose a multi-agent model, which can not only react to dynamic events, but can also generate schedules for maintenance jobs, to help design a fleet maintenance Decision Support System (DSS).

The remainder of this paper is organized as follows. Section 2 presents the description of the fleet maintenance problem. In Section 3, the MAS model for fleet maintenance scheduling is described, where the heuristic rules are put forward. The algorithm in which the dynamic problem is solved and schedules are generated is discussed in Section 4. Section 5 provides a case study of a mission oriented aircraft fleet to demonstrate the proposed method. Finally, concluding remarks and further study are provided in Section 6. 


\section{FLEET CBM PROBLEM DESCRIPTION}

Consider an aircraft fleet containing $m$ aircrafts and $n$ maintenance teams $(n<m)$ face continuous combat missions, in which a single mission requires $l$ aircrafts $(l$ is dynamic and $l \leq m$ ). Each aircraft contains $p L R M$ s whose $R U L$ can be estimated. All maintenance teams are of the same ability, namely the same $L R M$ requires the same Mean Maintenance Time (MMT), while different $L R M \mathrm{~s}$ require different $M M T \mathrm{~s}$. The basic assumptions of the problem are listed below.

1. The current mission is known, namely the upcoming mission and mission interval duration are known, while future missions are unknown.

2. Consider in-site maintenance only, so maintenance method is "replace and repair", and parts are repaired as good as new, namely the $R U L$ of replaced $L R M \mathrm{~s}$ reach the top.

3. The $R U L$ of each $L R M$ in each aircraft decreases with mission time, or $R U L$ doesn't decrease without a mission. Moreover, due to the differences in historical missions, the initial $R U L$ of different $L R M \mathrm{~s}$ in different aircrafts are different.

4. Spare parts in each team are sufficient, namely spare parts are always available whenever a maintenance task is required.

5. The estimation of $R U L$ is accurate, so the case in which wrong strategy led by wrong estimations won't occur.

6. Each team can work on only one aircraft at one time, and each aircraft can be repaired by only one team at one time.

After the whole fleet return from the previous mission, each aircraft checks its own health condition, estimating RULs and comparing the $R U L \mathrm{~s}$ with maintenance thresholds to decide a possible maintenance. There can be one or more threshold (Camci, Valentine \& Navarra, 2007), and in this article two thresholds are required, namely the Required Maintenance Threshold $\tau$ and the Opportunistic Maintenance Threshold $T$. Those two thresholds divide the aircraft into three health states. When $R U L \leqslant \tau$ the state is identified as the required maintenance state $S_{3}$ and a maintenance is required immediately. When $R U L>T$, the state is identified as the no maintenance state $S_{I}$ and no maintenance is scheduled. When $R U L$ is between these two thresholds $\tau<R U L \leqslant T$, the state is identified as the opportunistic maintenance state $S_{2}$ and a possible maintenance task depends on the states of other aircrafts and the occupation of maintenance teams. $T \& \tau$ can be set according to mission or by experience. For instance, $\tau$ must exceed the time before the aircraft returns from the next mission.

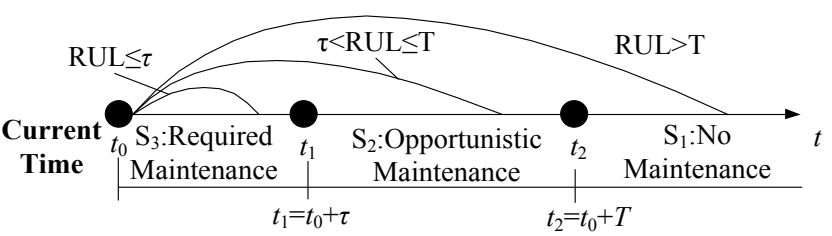

Figure 1. Maintenance thresholds and aircraft states

The objective of this problem is to maximize the availability of the fleet while the number of maintenance actions is satisfactory, and the basic constraints of the problem are:

1. The number of available aircrafts heading for the upcoming mission $r$ must satisfy $r \geqslant l$.

2. The number of currently available teams $s$ must satisfy $s \leqslant n$.

According to the description towards the problem above, when the fleet return from the previous mission, each aircraft checks its own health state $S_{t}$ at the current time $t_{0}$, and reports to the maintenance center. The maintenance center verifies all the reports, organizes and coordinates maintenance tasks guided by a set of heuristic rules, and allocate maintenance tasks to suitable maintenance teams. Maintenance teams then execute maintenance tasks according to the maintenance center. When a maintenance task finishes, the fleet wait to execute the upcoming mission.

Each aircraft in the fleet will be repaired according to its condition. To all aircrafts, the combination of all maintenance decisions within the whole fleet forms a group of fleet $C B M$ strategies aimed at utilizing the $R U L \mathrm{~s}$ of all aircrafts and the idle time of maintenance teams, in order to rationalize maintenance resources within the whole fleet.

\section{The Fleet CBM Model BaSed on Multi-Agent}

The fleet $C B M$ process involves a huge amount of communication among aircrafts, maintenance teams and the maintenance center. Moreover, maintenance teams and the maintenance center need to react to dynamic situations to make maintenance decisions and solve the problem, thus it can be regarded as a complex system (Zhang \& Li, 2010), and one promising solution to complex systems is $M A S$. In $M A S$, an agent can be regarded as a self-directed software object with its own value system and a means to communicate with other agents (Baker, 1998), while the whole $M A S$ can be regarded as "a loosely coupled network of problem solvers that work together to solve problems that are beyond the individual capabilities or knowledge of each problem solver" (Durfee, 1988). The fleet $C B M$ process can be mapped into a similar $M A S$, where $C B M$ strategies can be obtained via agents themselves and the communication between agents. 


\subsection{Model Framework}

Through the analysis of the fleet $C B M$ process, the physical entities can be abstracted into two types of agents, namely the Aircraft Agent $(A A)$ and the Maintenance Agent $(M A)$, and the dynamic process of management and coordination is abstracted into the Management and Coordination Agent $(M C A)$.

$A A$ is the abstract of an aircraft, it describes the inherent characteristics, the reliability characteristics, and is responsible for generating maintenance requirements. $M A$ is the abstract of maintenance teams, and is responsible for specific maintenance process.

$M C A$ is the abstract of the whole process of scheduling and intelligent allocating of maintenance tasks, it is driven by events, and is responsible for adjusting the whole process of maintenance, and obtaining the fleet maintenance strategy.

A 2-layer structure of $M A S$ (Feng, Zeng \& Kang, 2010) is applied to model the problem, each layer indicating the global scheduling and local scheduling, as shown in Figure 2 .

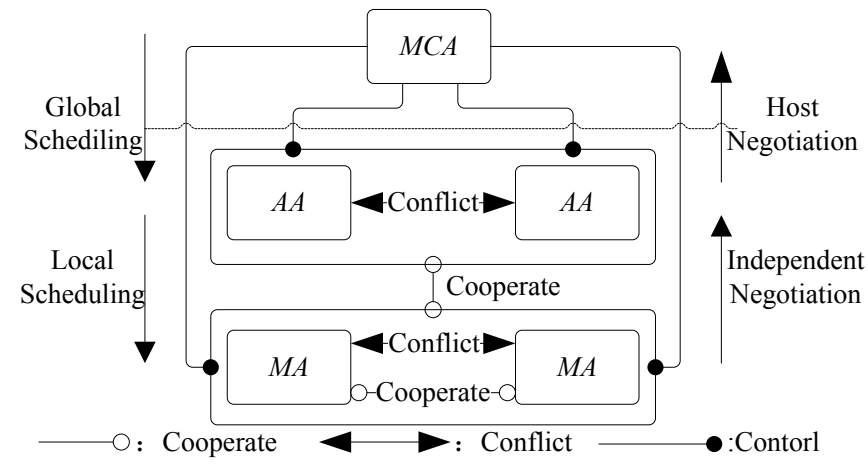

Figure 2. fleet $C B M M A S$ model framework

Global Scheduling is conducted by $M C A$. When $M C A$ receive the reports from $A A \mathrm{~s}$, it coordinates and controls the whole process and generates the overall maintenance strategy, to globally rationalize maintenance resources.

Local Scheduling is conducted between $A A \mathrm{~s}$ and $M A \mathrm{~s}$, aimed at the negotiation in specific maintenance tasks.

\subsection{Heuristic Rule-based Agent Negotiating Mechanism}

The Contract Net Protocol (CNP) (Smith, 1980) is one of the most widely used agent negotiating mechanisms. Through imitating the "Calling-Bidding-Winning-Signing" process in economic behavior, CNP realizes the allocation, dynamic adjusting and converting of tasks among agents (Tang, Zhu, Li \& Lei, 2010). Based on the CNP, the rationalization of the fleet $C B M$ strategies is achieved.

In this article, all agents are assumed rational and friendly, their communication manifest cooperation and conflicts, which means that an agent is willing to cooperate with other agents, and maximize its own profit if possible. That assumption caters for practical situations. For instance, each aircraft wishes to be repaired as early as possible. A maintenance team needs cooperation to repair all aircrafts, but wishes to repair as many aircrafts as possible.

Since the $M A S$ model applies the 2-layer structure, the negotiating between agents is also divided into two layers, namely the Host Negotiating and the Independent Negotiating. As proved above, the problem of a fleet maintenance with multiple maintenance teams is N-P hard, it's difficult to obtain the satisfying solution. So in each layer, negotiation must follow its corresponding heuristic rules, as described below.

\subsubsection{Heuristic Rules in Independent Negotiation}

In Independent Negotiation, idle $M A$ s communicate with $A A$ s to obtain local maintenance strategies, the alternative maintenance decision making heuristic rules are listed below.

1. Aircrafts in the required maintenance state $S_{3}$

- The shortest total waiting time principle: all aircrafts in the required maintenance state $S_{3}$ are scheduled to shorten the average waiting time, or to even the working time of all maintenance teams. This rule is marked "Rule 11a".

- The most repairs within limited interval principle: once a maintenance team is idle, a maintenance task is performed on the aircraft with the shortest $M M T$. This rule is marked "Rule $11 \mathrm{~b}$ ".

- $\quad$ Single team with widest repair time margin principle: as many aircrafts are repaired by as few maintenance teams as possible, so as to leave the most teams idle, in case unexpected failures occur. This rule is marked "Rule $11 \mathrm{c} "$.

2. Aircrafts in the opportunistic maintenance state $S_{2}$

- The most repairs within limited interval principle: once a maintenance team is idle, a maintenance task is performed on the aircraft with the shortest MMT. This rule is marked "Rule $12 \mathrm{a}$ ".

\subsubsection{Heuristic Rules in Host Negotiation}

In Host Negotiation, the $M C A$ communicates with $A A$ s to obtain global maintenance strategies, generates a group of local maintenance tasks and dispatches tasks to corresponding $M A \mathrm{~s}$. The whole process is listed below:

Assume that the number of aircrafts needed for the upcoming mission is $l_{n}$. AAs first report their health states $S_{t}$ to the $M C A$. The $M C A$ analyses all data reported and confirms the number of $A A \mathrm{~s}$ in the required maintenance state $S_{3} m_{3}$, the number of $A A \mathrm{~s}$ in the opportunistic maintenance state $S_{2} m_{2}$, and the number of $A A \mathrm{~s}$ in the no maintenance state $S_{l} m_{l}$. The $M C A$ then calculates the 
number of repairable aircrafts within the interval $m_{4}$ according to Rule $11 \mathrm{a}$, Rule $11 \mathrm{~b}$ and Rule $11 \mathrm{c}$ respectively, and gets the maximum number $m_{4}$, and the optimal rule is expressed as $\operatorname{Pro}\left(R u l_{i}\right)$. The number of combat-ready $A A \mathrm{~s}$ $m_{a}=m_{1}+m_{2}+m_{4}$. The alternative maintenance decision making heuristic rules are shown in Figure 3.

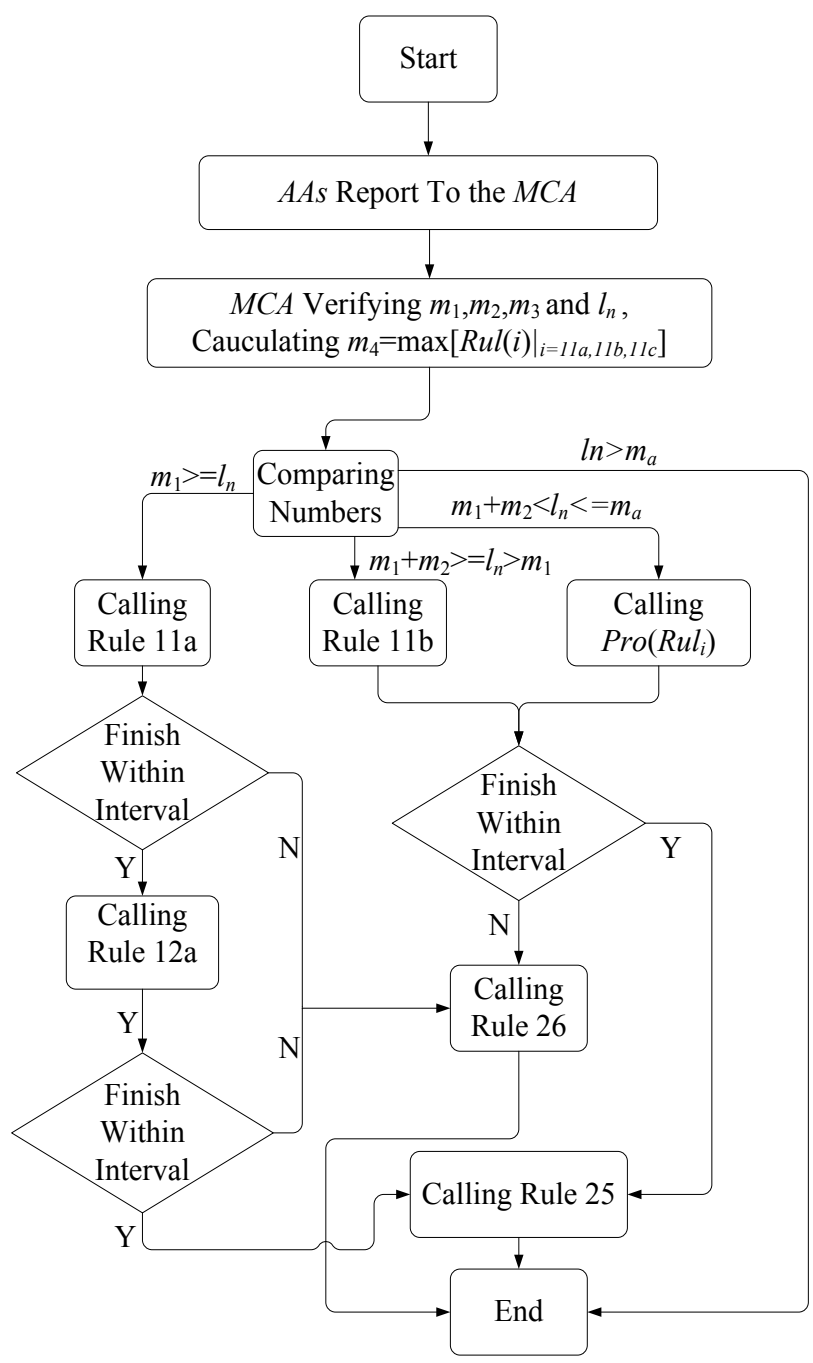

Figure 3. The flow chart of generating maintenance tasks

Based on the analysis above, the alternative maintenance decision making heuristic rules are listed below.

1. If $m_{a}<l_{n}$, then mission fails. This rule is marked "Rule $21 "$.

2. If the number of $A A \mathrm{~s}$ in the no maintenance state $S_{I}$ satisfies $m_{l} \geqslant l_{n}$, then $A A \mathrm{~s}$ in the no maintenance state $S_{I}$ are put on mission first, and $A A \mathrm{~s}$ in the required maintenance state $S_{3}$ are repaired according to Rule 11a. When current task finishes, $A A \mathrm{~s}$ in the opportunistic maintenance state $S_{2}$ are repaired according to Rule 12a, where $A A \mathrm{~s}$ with the shortest $M M T$ are repaired with high priority. This rule is marked "Rule 22".
3. If $m_{1}<l_{n} \leqslant m_{1}+m_{2}$, then $A A \mathrm{~s}$ in the required maintenance state $S_{3}$ are repaired according to Rule $11 \mathrm{~b}$. This rule is marked "Rule 23".

4. If $m_{1}+m_{2}<l_{n} \leqslant m_{1}+m_{2}+m_{4}$, then $A A \mathrm{~s}$ in the no maintenance state $S_{I}$ and the opportunistic maintenance state $S_{2}$ are put on mission first, and $A A \mathrm{~s}$ in the required maintenance state $S_{3}$ are repaired according to $\operatorname{Pro}\left(R u l_{i}\right)$. This rule is marked "Rule 24".

5. When the interval ends, each aircraft checks its health state again, and reports to the $M C A$. Then the $M C A$ analyses the reported data and select $l_{n} A A \mathrm{~s}$ with the shortest $R U L$ out of all combat-ready $A A \mathrm{~s}$ ( $A A \mathrm{~s}$ in the opportunistic maintenance state $S_{2}, A A \mathrm{~s}$ in the no maintenance state $S_{I}$ and repaired $A A \mathrm{~s}$ ) to execute the mission. This rule is marked "Rule 25".

6. When mission starts, if there exists still $A A \mathrm{~s}$ in the opportunistic maintenance state $S_{2}$ required maintenance state $S_{3}$ among all the left-over $A A$ s, then those $A A \mathrm{~s}$ are repaired according to Rule 11a and Rule 12a respectively. This rule is marked "Rule 26".

\subsubsection{Agent Behavior in fleet CBM}

Based on the analysis of the process of fleet $C B M$, the $M A S$ model framework and the heuristic rules on solving maintenance strategies, the Agent Ability Chart (Feng, 2009) in fleet $C B M$ is established, which finally defines agents' attributes and behaviors of function \& fault, laying the foundation of solving the maintenance strategies.

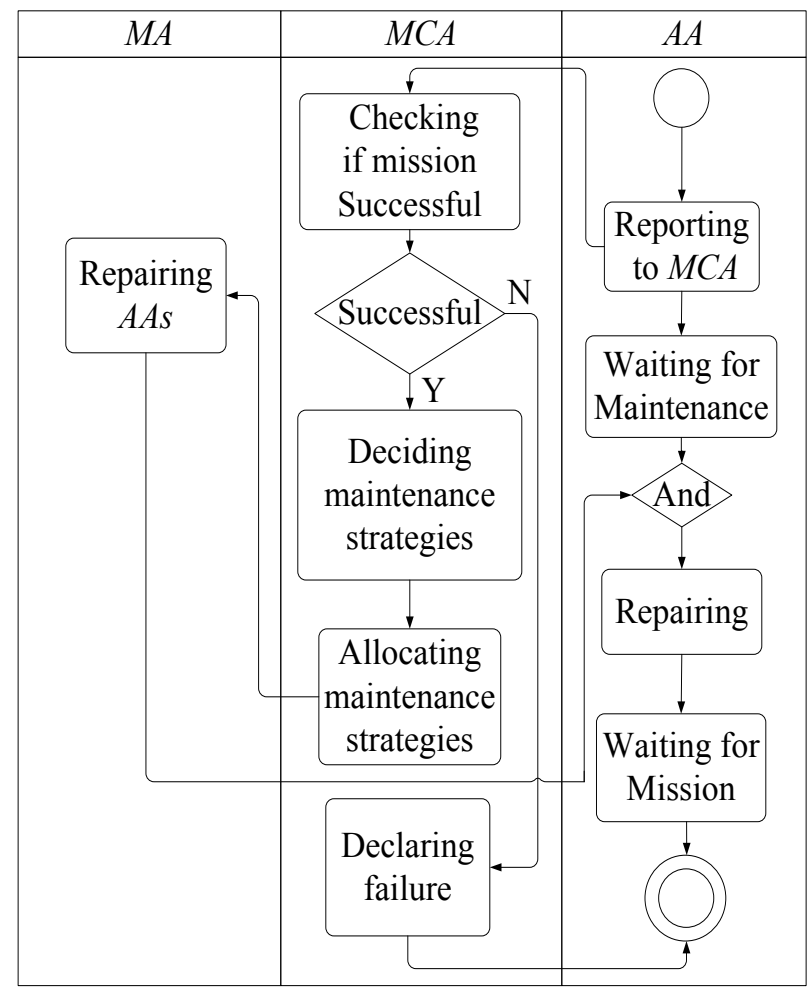

Figure 4. The Agent ability chart in fleet $C B M$ 


\section{Maintenance Strategy Solving Algorithm}

AUML (Bauer, Müller \& Odel, 2001), based on object oriented design, is a typical agent oriented modeling technique. It provides a uniform agent oriented modeling mechanism, and doesn't restrict too much on modeling process. The process of fleet $C B M$ can be realized through the communication between $A A \mathrm{~s}$ and $M A \mathrm{~s}$. With the help of AUML, the negotiating models between agents are established based on $C N P$.

Since the $C B M$ model involves communication between and within layers, the problem is relatively complex. As space is limited, three of the most typical maintenance schemes are illustrated. These three corresponding algorithms are listed below.

\subsection{The Shortest Total Maintenance Waiting Time Maintenance Scheme Negotiating Algorithm}

This scheme is relatively integrated, which involves cooperative and competitive negotiations. The algorithm is listed below.

\subsubsection{Cooperative Negotiation}

Cooperative negotiation is required before a maintenance task starts. It's aimed at calculating the whole maintenance time needed and allocating each $M A$ its corresponding maintenance time.

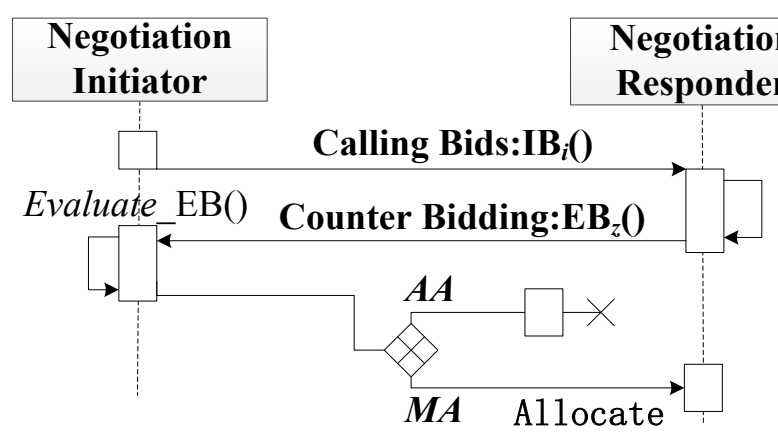

Figure 5. The cooperative negotiation mechanism

Step 1: The negotiation initiator calling for bids.

The first idle $M A i$ (a random $M A$ if there exists more than one) calls other $M A \mathrm{~s}$ and all $A A \mathrm{~s}$ for bids $I B_{i}\left(t_{i} \mid t a_{i}, t b_{i}\right)$, where $t_{i}$ represents the latest bid time allowed, $t a_{i}$ represents the earliest idle time of other $M A$ s (Time to finish current task), $t b_{i}$ represents the maintenance duration needed.

Step 2: The negotiation responders counter-bidding.

$M A$ s and $A A$ s assess their own status and counter-bid before $t_{i}$. The counter-bids from $M A \mathrm{~s}$ are represented as $E B_{j}\left(t_{j} \mid t a_{j}\right)$, where $t_{j}$ represents the waiting time, $t a_{j}$ represents the earliest idle time. While the counter-bids from $A A$ s are represented as $E B_{k}\left(t_{k} \mid t b_{k}\right)$, where $t_{k}$ represents the waiting time, $t a_{k}$ represents the maintenance time needed.

Step 3: The negotiation initiator responding to all counterbids

The negotiation initiating $M A$ counts all counter-bids. Assume that $m$ is the number of counter-bids from $M A$ s and $n$ is the number of counter-bids from $A A$ s. Then based on the Shortest Total Maintenance Waiting Time Principle, The negotiation initiating $M A$ calculates the Allocated Maintenance Time (AMT) to $M A j$ through function Evaluate_EB(),

$$
A M T_{j}=\left(\sum_{1}^{m} t a_{j}+\sum_{1}^{n} t b_{k}\right) / m-t a_{j}
$$

and responds to each $M A$ its $A M T$.

\subsubsection{Competitive Negotiation}

Competitive negotiation is required during the process of specific maintenance tasks. It's aimed at confirming maintenance strategy and realizing maintenance tasks.

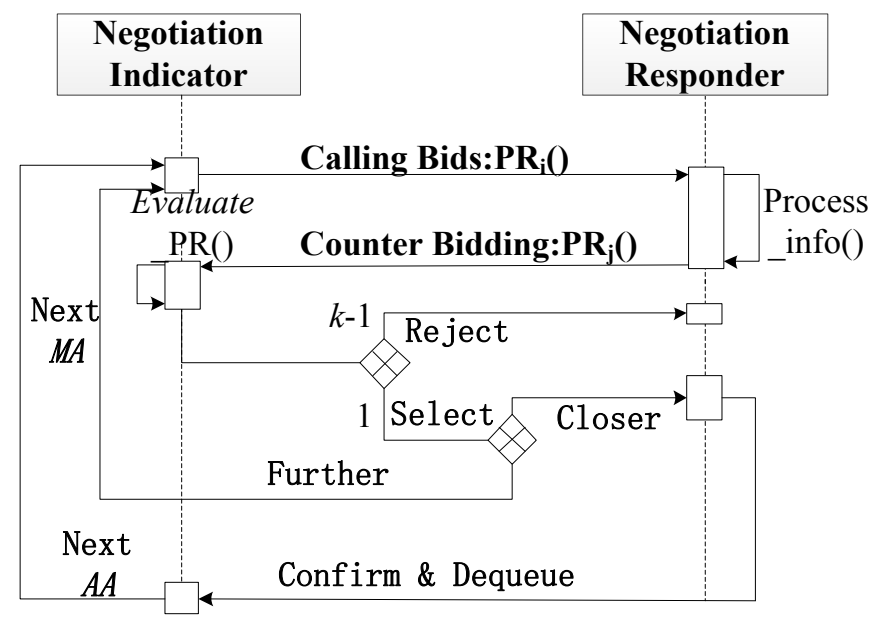

Figure 6 . The competitive negotiation mechanism

Step 1: $M A$ calling for bids.

The first idle $M A i$ (a random $M A$ if there exists more than one) calls all $A A \mathrm{~s}$ for bids $P R_{i}\left(T_{i} \mid A M T_{i}\right)$, where $T_{i}$ represents the latest bid time allowed, $A M T_{i}$ represents the allocated maintenance time.

Step 2: AAs counter-bidding.

AAs assess their own status through function Process_info(). If it's within the candidate queue, then counter-bid before $t_{i}$. The counter-bids from $A A \mathrm{~s}$ are represented as $P R_{j}\left(T_{j} \mid M M T_{j}\right)$, where $T_{j}$ represents the waiting time, $M M T_{j}$ represents the maintenance time needed. 


\section{Step 3: $M A$ assessing all counter-bids}

$M A$ counts all counter-bids and assesses them through function Evaluate $E B($ ), ranking all counter-bidding $A A \mathrm{~s}$ according to the length of $M M T$ and selecting the candidate $a$ with the closest $M M T$ to $A M T$.

Step 4: $M A$ judging whether to stop bidding.

$M A$ updates its $A M T: A M T_{\text {temp }}=A M T-M M T_{a}$ for the moment. If abs $\left(A M T_{\text {temp }}\right)<$ abs $(A M T)$, then $M A$ updates the $A M T=A M T_{\text {temp }}$ and responds to the selected $A A$ and the selected $A A$ then dequeues, repeat Step1 $\sim$ Step3. Else, $M A$ stops the current process of bidding and starts repairing all selected $A A$ s.

Step 5: Other $M A$ s start bidding according to the idle time order (a random $M A$ if there exists more than one), repeat Step1 $\sim$ Step4.

\subsection{The Most Repairs Within the Limited Interval Maintenance Scheme Negotiating Algorithm}

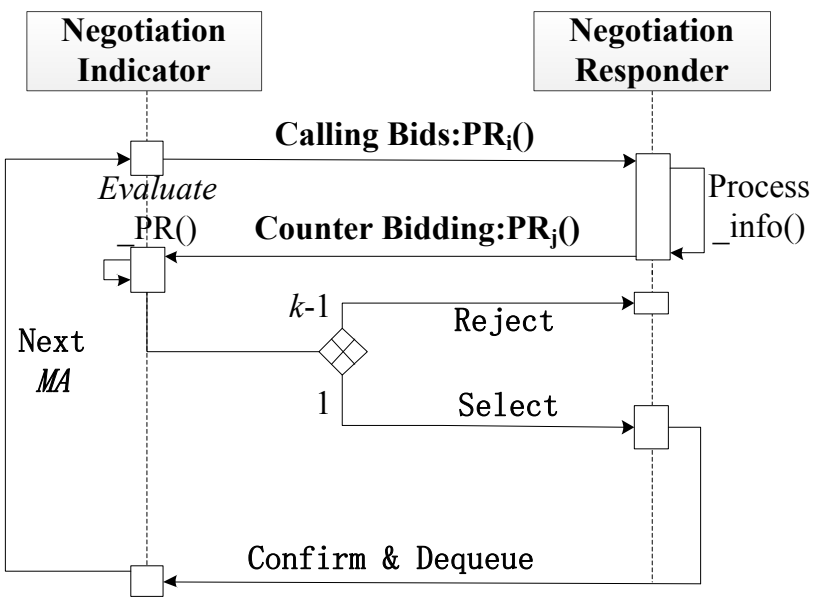

Figure 7. The most repairs within the limited interval maintenance scheme negotiation mechanism

Step 1: $M A$ calling for bids.

The first idle $M A i$ (a random $M A$ if there exists more than one) calls all $A A \mathrm{~s}$ for bids $P R_{i}\left(T_{i} \mid L M T_{i}\right)$, where $T_{i}$ represents the latest bid time allowed, $L M T_{i}$ represents the longest maintenance time.

Step 2: $A A$ s counter-bidding.

AAs assess their own status through function Process_info(). If it's within the candidate queue, then counter-bid before $t_{i}$. The counter-bids from $A A \mathrm{~s}$ are represented as $P R_{j}\left(T_{j} \mid M M T_{j}\right)$, where $T_{j}$ represents the waiting time, $M M T_{j}$ represents the maintenance time needed.

Step 3: $M A$ assessing all counter-bids
$M A$ counts all counter-bids and assesses them through function Evaluate_EB(), ranking all counter-bidding $A A \mathrm{~s}$ according to the length of $M M T$ and selecting the candidate $a$ with the shortest $M M T$. Then repair task starts, when task finishes, $M A$ updates its $L M T=L M T-M M T_{a}$.

Step 4: The repaired $A A$ then dequeues. Other $M A$ s start bidding according to the idle time order (a random $M A$ if there exists more than one), repeat Step1 $\sim$ Step3.

\subsection{Single Team with Widest Repair Time Margin Maintenance Scheme Negotiating Algorithm}

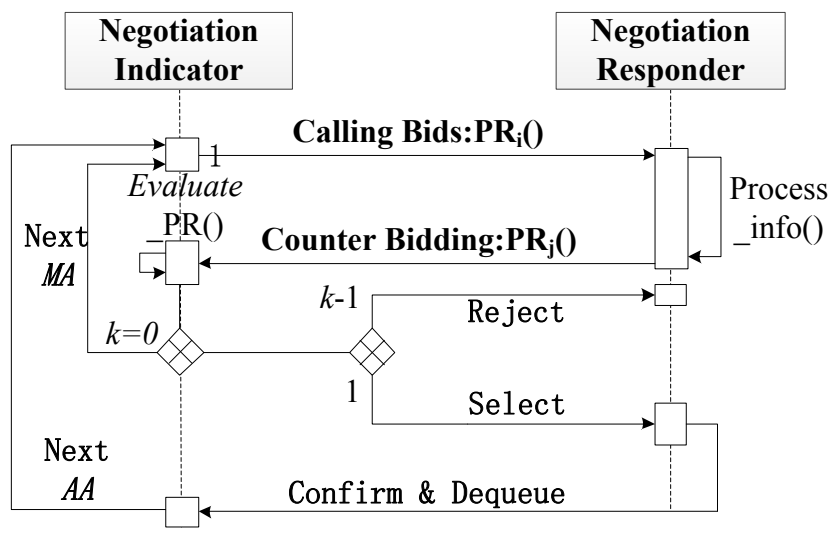

Figure 8 . Single team with widest repair time margin maintenance scheme negotiation mechanism

Step 1: $M A$ calling for bids.

The first idle $M A i$ (a random $M A$ if there exists more than one) calls all $A A \mathrm{~s}$ for bids $P R_{i}\left(T_{i} \mid L M T_{i}\right)$, where $T_{i}$ represents the latest bid time allowed, $L M T_{i}$ represents the longest maintenance time.

Step 2: AAs counter-bidding.

$A A \mathrm{~s}$ assess their own status through function Process_info(). If it's within the candidate queue, then counter-bid before $t_{i}$. The counter-bids from $A A \mathrm{~s}$ are represented as $P R_{j}\left(T_{j} \mid M M T_{j}\right)$, where $T_{j}$ represents the waiting time, $M M T_{j}$ represents the maintenance time needed.

Step 3: $M A$ assessing all counter-bids

$M A$ counts all counter-bids and assesses them through function Evaluate_EB(), ranking all counter-bidding $A A \mathrm{~s}$ according to the length of $M M T$ and selecting the candidate $a$ with the closest $M M T$ to $L M T$. Then the selected $A A$ dequeues.

Step 4: $M A$ updates its $L M T=L M T-M M T_{a}$ and repeats Step1 $\sim$ Step3, till there's no suitable candidate. Then stop bidding and start repairing all selected $A A$ s. 
Step 5: Other $M A$ s start bidding according to the idle time order (a random $M A$ if there exists more than one), repeat Step1 Step4.

\section{CASE STUdY}

A typical continuous mission of a fleet is presented to verify the proposed fleet $C B M$ decision making strategy. Assume a fleet consisting of 10 aircrafts, each monitoring the condition of two LRMs and predicting their corresponding $R U L s$, which carries on a 3-wave mission. The time property of the mission is listed in Table 1

\begin{tabular}{l|l|l|l}
\hline No. & $\begin{array}{l}\text { Start Time } \\
(\boldsymbol{h})\end{array}$ & $\begin{array}{l}\text { Mission } \\
(\boldsymbol{h})\end{array}$ & $\begin{array}{l}\text { Interval } \\
(\boldsymbol{h})\end{array}$ \\
\hline 1 & 1 & 2 & 2 \\
\hline 2 & 5 & 3 & 1 \\
\hline 3 & 9 & 2.5 & - \\
\hline
\end{tabular}

Table 1. Time property of the mission

During the mission, there exist two maintenance teams to support the whole fleet. The maintainability $(M M T)$ of the two LRMs are listed in Table 2

\begin{tabular}{l|l}
\hline LRM & MMT \\
Sorts & $(\boldsymbol{h})$ \\
\hline$L R M_{1}$ & 0.5 \\
\hline$L R M_{2}$ & 1 \\
\hline
\end{tabular}

Table 2. Reliability and maintainability data

To effectively verify this method, assume that some aircrafts are in the no maintenance state $S_{I}$ while others are in the opportunistic maintenance state $S_{2}$, hence all aircrafts can take part in the first mission, and no maintenance is considered before the first wave. The initial $R U L$ of all aircrafts in the fleet are listed in Table 3

\begin{tabular}{l|l|l|l|l|l}
\hline ARM & & 2 & 3 & 4 & 5 \\
\hline$L R M_{1}$ & 16.3 & 8.5 & 12.6 & 5.6 & 19.1 \\
\hline$L R M_{2}$ & 18.1 & 18.2 & 5.9 & 10.9 & 19.2 \\
\hline
\end{tabular}

\begin{tabular}{l|l|l|l|l|l}
\hline ARM & & 7 & 8 & 9 & 10 \\
\hline$L R M_{1}$ & 5.1 & 19.1 & 16.0 & 8.4 & 15.8 \\
\hline$L R M_{2}$ & 19.4 & 9.7 & 5.8 & 18.3 & 19.1 \\
\hline
\end{tabular}

Table 3. The initial $R U L$ of the fleet

Since the future missions are unknown, the maintenance thresholds can be decided as: $\tau$ is the time before the aircraft return from the next mission, and $T$ is $T=2 \times \tau$. On each inspection, the maintenance thresholds are listed in Table 4

\begin{tabular}{c|l|l}
\hline $\begin{array}{l}\text { Maintenance } \\
\text { thresholds }(\boldsymbol{h})\end{array}$ & $\begin{array}{l}\text { First } \\
\text { Inspection }\end{array}$ & $\begin{array}{l}\text { Second } \\
\text { Inspection }\end{array}$ \\
\hline$\tau$ & 3 & 2.5 \\
\hline$T$ & 6 & 5 \\
\hline
\end{tabular}

Table 4. Maintenance thresholds on each inspection

Assume that the first wave requires 8 aircrafts, the second wave requires 6 and the third wave requires all aircrafts. Based on our former assumptions and the maintenance decision making rules, maintenance strategies can be obtained as listed in Table 5, where the 2nd and 3rd column indicates the number of aircrafts repaired in the team. For instance, "3,4" means aircraft 3 and 4 are repaired in team 1. The 4th column indicates the spared aircrafts from the mission, for instance, "1,5" indicates aircraft 1 and 5 are spared from this mission. The 5 th column indicates whether this mission is successful.

\begin{tabular}{l|l|l|l|l}
\hline Waves & $\begin{array}{l}\text { Queue } \\
\mathbf{1}\end{array}$ & $\begin{array}{l}\text { Queue } \\
\mathbf{2}\end{array}$ & Spared & $\begin{array}{l}\text { Mission } \\
\text { Succeed }\end{array}$ \\
\hline 1 & null & null & 1,5 & $\mathrm{Y}$ \\
\hline 2 & 3,4 & 6,8 & $1,5,6,8$ & $\mathrm{Y}$ \\
\hline 3 & 2,9 & 7 & null & $\mathrm{Y}$ \\
\hline
\end{tabular}

Table 5. Fleet maintenance strategies.

Traditional CBM methods, which concentrates more on "timely" maintenance decision making rather than "online", can hardly make maintenance decisions online, so is not comparable with the $M A S$ method. To make the comparison possible, $M A S$ is applied to model traditional $C B M$ policy, which assumes that an aircraft is repaired only when it comes to the required maintenance state $S_{3}$, relies on a single threshold, and ignores the states of the whole fleet and the maintainability of limited teams. Assume that the initial state, mission time property and maintainability of teams are the same, and the fleet maintenance strategies are listed in Table 6

\begin{tabular}{l|l|l|l|l}
\hline Waves & $\begin{array}{l}\text { Queue } \\
\text { 1 }\end{array}$ & $\begin{array}{l}\text { Queue } \\
\mathbf{2}\end{array}$ & Spared & $\begin{array}{l}\text { Mission } \\
\text { Succeed }\end{array}$ \\
\hline 1 & null & null & 1,5 & Y \\
\hline 2 & null & null & $1,5,6,10$ & Y \\
\hline 3 & 3 & 4,6 & 8 & $\mathrm{~N}$ \\
\hline
\end{tabular}

Table 6. Single threshold fleet maintenance strategies

The table shows that before the 3rd wave, aircraft 3,4,6,8 all need repairing, and the total time required is $3 h$, which exceeds the maximum time teams can offer, so mission fails.

The case above shows that the 2-thresholds $C B M$ policy is superior to traditional single-threshold $C B M$ policy in both flexibility and results. 


\section{CONCLUSION}

In this paper, a fleet $C B M$ intelligent decision making method based on MAS and heuristic rules is proposed, which is a technical support for fleet online maintenance decision making, and can help design a fleet maintenance Decision Support System (DSS). A fleet consisting of 10 aircrafts and 2 teams is illustrated to verify the correctness and feasibility of this method.

To avoid the local optimal solution, host negotiating is proposed to coordinate the global maintenance strategies, which can not only guarantee the correctness and feasibility of the solution, but also optimize the global maintenance strategy.

A 2-thresholds $C B M$ policy is proposed, and results show that the 2-thresholds $C B M$ policy is superior to traditional single-threshold $C B M$ policy in both flexibility and results, while the requirement to decide maintenance threshold is much higher.

This method mainly concentrates on the strategy itself. With suitable improvement, this method can be modified to optimize maintenance resources.

This method is based on an assumption that the $R U L$ estimation is accurate, and the maintenance strategies are based on accurate RULs. Considering the defects in failure prognostics technology, further study needs to discuss the relationship between the accuracy of the $R U L$ estimates and the availability of the fleet, where $P H M$ uncertainty management will be considered.

\section{ACKNOWLEDGEMENT}

This work is partially supported by the Fundamental Research Funds for the Central Universities of China (No. YWF-12-LSJC-001).

\section{NOMENCLATURE}

$\begin{array}{ll}A A & \text { Aircraft Agent } \\ A M T & \text { Allocated Maintenance Time } \\ C B M & \text { Condition-Based Maintenance } \\ C N P & \text { Contract Net Protocol } \\ L M T & \text { Longest Maintenance Time } \\ L R M & \text { Line Replaceable Module } \\ M A & \text { Maintenance Agent } \\ M A S & \text { Multi-Agent System } \\ M C A & \text { Management and Coordination Agent } \\ M M T & \text { Mean Maintenance Time } \\ P H M & \text { Prognostics and Health Management } \\ R U L & \text { Remaining Useful Life }\end{array}$

\section{REFERENCES}

Jiang, R. Y. \& Murthy, D. N. P. (2008). Maintenance: Decision Models for Management. Beijing: Science Press.

Bengtsson, M. (2004). Condition Based Maintenance System Technology - Where is Development Heading. Congress Report on the $17^{\text {th }}$ Euromaintenance, May, Barcelona, Spain. http://www.idp.mdh.se/forskning/amnen/produktproces $\mathrm{s} /$ projekt $/ \mathrm{cbm} /$ publi-

kationer/em04\%20paper\%20Marcus\%20Bengtsson.pdf.

Sun, B., Zeng, S.K., Kang, R. \& Pecht, M. G. (2012). Benefits and Challenges of System Prognostics. Reliability. IEEE Transactions on, vol. 61(2), pp. 323335. doi:10.1109/TR.2012.2194173

Goebel, K.F., Saha B., Saxena, A., Celaya, J.R. \& Christophersen, J.P. (2008). Prognostics in Battery Health Management. Instrumentation \& Measurement Magazine, IEEE, vol. 11(4), pp. 33-40. doi:10.1109/MIM.2008.4579269

Wen, Z.H. \& Liu, Y.P. (2011). Applications of Prognostics and Health Management in aviation industry. Prognostics and System Health Management Conference. May 24-25, Shenzhen, China. doi:10.1109/PHM.2011.5939539

Barata, J., Guedes, S.C., Marseguerra, M. \& Zio, E. (2002). Simulation modeling of repairable multi-component deteriorating systems for 'on condition' maintenance optimization. Reliability Engineering \& System Safety, vol. 76(3), pp. 255-264. doi:10.1016/S09518320(02)00017-0

Jardine, A.K.S., Lin, D. \& Banjevic, D. (2006). A review on machinery diagnostics and prognostics implementing condition-based maintenance. Mechanical Systems and Signal Processing, vol. 20(7), pp. 1483-1510. doi:10.1016/j.ymssp.2005.09.012

Garey, M.R. \& Johnson, D.S. (1979). Computers and Intractability: A Guide to the Theory of NPCompleteness. New York, USA: WH Freeman \& Co.

Doganay, K. \& Bohlin, M. (2010). Maintenance plan optimization for a train fleet. WIT Transactions on The Built Environment, vol. 114(12), pp. 349-358. doi:10.2495/CR100331

Bai, F. (2009). Methods of Scheduling and Condition Based Mainteance Decision Making in Civil Aero Engine Fleet. Doctoral dissertation. Nanjing University of Aeronautics and Astronautics, Nanjing, China

Reimann, J., Kacprzynski, G., Cabral, D. \& Marini, R. (2009). Using Condition Based Maintenance to Improve the Profitability of Performance Based Logistic Contracts. Annual Conference of the Prognostics and Health Management Society, Sep 27Oct 1, San Diego, California. http://www.phmsociety.org/sites/phmsociety.org/files/p hm_submission/2009/phmc_09_52.pdf

Bivona, E. \& Montemaggiore, G.B. (2005). Evaluating Fleet and Maintenance Management Strategies through 
System Dynamics Model in a City Bus Company. The 23rd International Conference of the System Dynamics Society, July 17-21, Boston. http://www.systemdynamics.org/conferences/2005/proc eed/papers/MONTE431.pdf

Dupuy, M.J., Wesely, D.E. \& Jenkins, C.S. (2011). Airline fleet maintenance: Trade-off analysis of alternate aircraft maintenance approaches. Systems and Information Engineering Design Symposium (SIEDS). April 29, Charlottesville, VA. doi:10.1109/SIEDS.2011.5876850

Cycon, J.P. (2011). The journey to incorporate health monitoring and condition based maintenance of Sikorsky commercial helicopters. 8th International Workshop on Structural Health Monitoring 2011: Condition-Based Maintenance and Intelligent Structures, September 13-15, Stanford, CA. http://www.destechpub.com/links/catalogs/bookstore/ad vanced-materials-sciencetechnology/structural-healthmonitoring/structural-health-monitoring-2011/\#table

Zhou, R., Fox, B., Lee, H.P. \& Nee., A.Y.C. (2004). Bus maintenance scheduling using multi-agent systems. Engineering Applications of Artificial Intelligence, vol. 17(6), pp. 623-630. doi:10.1016/j.engappai.2004.08.007

Papakostas, N., Papachatzakis, P., Xanthakis, V., Mourtzis, D. \& Chryssolouris, G. (2010). An approach to operational aircraft maintenance planning. Decision Support Systems, vol. 48(4), pp. 604-612. doi:10.1016/j.dss.2009.11.

Budenske J., Newhouse J., Bonney J. \& Wu J. (2001). Agent-based schedule validation and verification. Systems, Man, and Cybernetics, 2001 IEEE International Conference on. Oct 7-10, Tucson, AZ. doi:10.1109/ICSMC.2001.969920

Yang, S.L. \& Hu, X.J. (2007). Complex decision task modeling and solving methods. Beijing: Science Press

Camci, F., Valentine, G.S. \& Navarra, K. (2007). Methodologies for Integration of PHM Systems with Maintenance Data. Aerospace Conference, 2007 IEEE, March 3-10, Big Sky, MT. doi:10.1109/AERO.2007.352917

Zhang, G.J. \& Li, Y.D. (2010). Agent-based modeling and simulation for open complex systems. Informatics in Control, Automation and Robotics (CAR), 2010 2nd International Asia Conference on, March 6-7, Wuhan. doi: 10.1109/CAR.2010.5456783

Baker, A.D. (1998). A survey of factory control algorithms that can be implemented in a multi-agent heterarchy: dispatching, scheduling and pull. Journal of Manufacturing Systems, vol. 17(4), pp. 297-320. doi: 10.1016/S0278-6125(98)80077-0

Durfee, E.H. (1988). Coordination of Distributed Problem Solvers. Boston, MA: Kluwer Academic Publishers.

Feng, Q, Zeng, S.K. \& Kang, R. (2010) Multiagent-based modeling method for integrated logistic support of the carrier aircraft. Systems Engineering and Electronics, vol. 32(1), pp. 211-217.

Smith, R.G. (1980). The Contract Net Protocol: High-Level Communication and Control in a Distributed Problem Solver. Computers, IEEE Transactions on, vol. C29(12), pp. 1104-1113. doi: 10.1109/TC.1980.1675516

Tang, S.Y., Zhu, Y.F., Li, Q. \& Lei, Y.L. (2010). Survey of task allocation in multi Agent systems. Systems Engineering and Electronics, vol. 32(10), pp. 21552161. doi:0.3969/j/issn/1001-506X.2010.10.30

Feng, Q. (2009). Mesuring and Modeling and Optimization Method of Effectiveness for Complex Materiel System based on Multi-Agent. Doctoral dissertation. Beihang University, Beijing, China

Bauer, B., Müller, J.P. \& Odell, J. (2001). Agent UML: a formalism for specifying multiagent software systems. International Journal of Software Engineering and Knowledge Engineering, vol. 11(3), pp. 1-12. doi:10.1142/S0218194001000517

\section{BIOGRAPHIES}

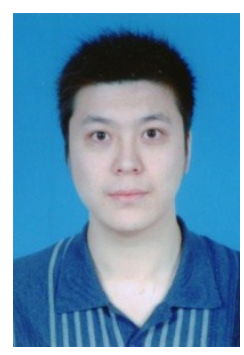

Dr. Qiang Feng is a reliability engineer and a member of the faculty of systems engineering at the School of Reliability and Systems Engineering at Beihang University in Beijing, China. He received his $\mathrm{Ph} . \mathrm{D}$. degree in reliability engineering and systems engineering from Beihang University and a B.S. degree in mechanical engineering from Beihang University. He has won a 1st and a 3rd prize for National Defense Science and Technology Progress Award. His current research interests include reliability engineering, reliability of complex product and integrated design of product reliability and performance.

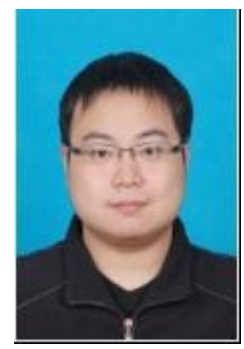

Songjie $\mathbf{L i}$ is a graduate student at the School of Reliability and Systems Engineering at Beihang University in Beijing, China. He received a B.S. degree in mathematics and control theory from Beihang University and is a master candidate. He has won a 2nd prize in Graduate Scholarship twice in a row. His current research interests include reliability engineering, reliability and performance of complex product and system modeling \& simulation.

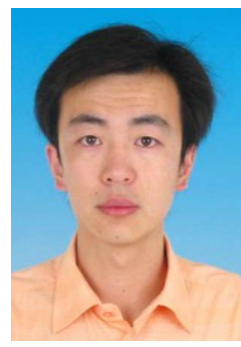

Dr. Bo Sun is a reliability engineer and a member of the faculty of systems engineering at the School of Reliability and Systems Engineering at Beihang University in Beijing, China. He received his $\mathrm{Ph}$.D. degree in reliability engineering and systems engineering from Beihang University and a B.S. degree in 
mechanical engineering from the Beijing Institute of Mechanical Industry. His current research interests include prognostics and health management, physics of failure, reliability of electronics, reliability engineering, and integrated design of product reliability and performance. $\mathrm{He}$ has won a 1st and a 3rd prize for National Defense Science and Technology Progress Award. He has published over 40 papers and 2 book chapters (Reliability Design and Analysis, and Diagnostics, Prognostics, and System's Health Management). He is now a member of the Editorial Board 